\title{
L'apport des méthodes de reconnaissance sismique à la reconnaissance des barrages en maçonnerie
}

\section{Contribution of geophysical methods to masonry dam assessment}

\author{
Y. BERTRAND \\ SEGG* \\ P. ROYET, G. DEGOUTTE \\ CEMAGREF $^{* *}$
}

Rev. Franç. Géotech. n 64, pp. $45-55$ (juillet 1993)

\section{Résumé}

La majeure partie des plus vieux barrages français sont des ouvrages en maçonnerie. La nécessité de mieux connaître leur état de santé et les phénomènes de vieillissement qui les affectent, se traduit par un important programme de reconnaissance qui a d'abord concerné les plus grands de ces barrages et qui est maintenant orienté vers des barrages de taille plus modeste.

Cet article présente une méthodologie générale adoptée pour la reconnaissance approfondie de tels ouvrages et décrit plus particulièrement l'apport des méthodes sismiques à partir des cas des barrages de la Bourne (26) et de la Gimond (69). Les diverses techniques de transparence sismique sont décrites de façon détaillée et, en particulier, leur adaptation à la reconnaissance des barrages en maçonnerie.

Les enseignements tirés des deux exemples traités amènent à proposer une méthodologie originale pour des reconnaissances futures sur d'autres barrages.

\section{Abstract}

Most of the oldest dams in France are masonry structures. The need to assess their current condition and ageing involves an extensive field investigation programme which commenced with the largest structures and is now progressing towards the smaller ones.

The article describes the general approach adopted for the detailed investigation of these dams, with emphasis on the contribution of seismic methods, using Bourne and Gimont dams as examples. Seismic transparency techniques are described in detail with reference to their suitability for masonry dam investigations.

From experience gained at these two dams, the authors propose an innovative methodology for future investigations on other dams.

\footnotetext{
* Société d'Etudes Géotechniques et Géophysiques, 8, av. Newton, 92350 Le Plessis-Robinson.

* CEMAGREF, groupement d'Aix-en-Provence, BP 31, Le Tholonet, 13612 Aix-en-Provence Cedex 1.
} 


\section{INTRODUCTION}

La nécessité de bien connaître et de surveiller les barrages anciens a conduit ces dernières années à la révision spéciale des plus grands des barrages français en maçonnerie construits avant le milieu du XX $\mathrm{X}^{e}$ siècle. Cependant l'état de nombreux barrages en maçonnerie reste encore mal connu, et leurs propriêtaires soupçonnent rarement les phénomènes de vieillissement qui les affectent.

Tout en laissant délibérément de côté les aspects hy. drologiques et hydrauliques, cet article présente une méthodologie générale adoptée pour la reconnaissance approfondie de tels ouvrages et décrit plus particulièrement l'apport original des méthodes sismiques à partir des cas des barrages de la Boume (26) et de la Gimond (69).

Les diverses techniques de transparence sismique sont décrites de façon détaillée et, en particulier, leur adaptation à la reconnaissance des barrages en maçonnerie.

\section{LE DIAGNOSTIC DES BARRAGES ANCIENS EN MAÇONNERIE}

\subsection{Des ouvrages souvent mal connus}

Depuis le début du XIX ${ }^{e}$ siècle, de nombreux barrages destinés à l'alimentation en eau ou à la production électrique ont été construits en France. Jusque vers 1930, la plupart de ces barrages étaient des ouvrages-poids en maçonnerie, le plus souvent légèrement arqués. Les plus grands d'entre eux, exploités par des organismes spécialisés ont fait l'objet de révisions spéciales qui ont parfois conduit à des travaux importants. Ils sont désormais bien connus et dotés de dispositifs d'auscultation adaptés.

En revanche, nombre de barrages anciens de dimension moyenne appartiennent à des maîtres d'ouvrages dispersés, ne possédant qu'un ou deux ouvrages et ne disposant pas de compétences particulières pour leur suivi. Vu leur âge respectable, ces barrages sont considérés comme faisant partie du paysage, et les phénomènes liés à leur vieillissement sont le plus souvent sous-estimés, voire ignorés.

Malheureusement, l'expérience montre que les maçonneries des barrages anciens vieillissent sous l'effet des sollicitations prolongées tant hydrauliques que mécaniques (LINO et al., 1991) :

- lente dissolution des liants due aux percolations de l'eau, entraînant à la fois une perte de poids de l'ouvrage, une augmentation des sous-pressions internes et une accélération des fuites;

- fatigue de la maçonnerie sous l'effet des déforma. tions répétées dues aux variations thermiques et aux fluctuations du plan d'eau.

Ce vieillissement des maçonneries ne se constate pas forcément du premier coup d'œeil, car les vieux ouvrages d'art en maçonnerie présentent des parements d'excellente qualité mais un remplissage plus médiocre (DE. GOUTTE, 1991).

L'évolution des fuites est aussi très difficile à apprécier, car ces barrages ont rarement été dotés d'un dispositif fiable de mesure des débits et, de toutes façons, les percolations à la base de l'ouvrage ne sont pas obser. vables.

En outre, nombre de barrages anciens ont été surélevés pour faire face à une augmentation des besoins en eau, sans que ces travaux aient forcément été justifiés par une étude de stabilité. Cela ne fait bien sûr qu'accroître le risque encouru et renforce la nécessité d'un dia. gnostic approfondi.

Pour toutes ces raisons, il importe de se donner tous les moyens pour connaître la constitution des vieux barrages.

\subsection{Les techniques de reconnaissance des barrages en maçonnerie}

La reconnaissance des barrages en maçonnerie repose essentiellement sur des sondages carottés prolongés en fondation qui permettent:

- la mesure des paramètres de forage ou diagraphies instantanées (pression sur l'outil, couple de rotation, vitesse d'avancement) :

- l'observation des carottes et la détermination de l'état de fracturation du matériau prélevé:

- le choix d'échantillons intacts pour des mesures de densité, porosité et résistance mécanique;

- l'observation des parois du forage par caméra vidéo, lorsque le carottage est très médiocre;

- la réalisation d'essais de perméabilité en forages (type Lugeon) dans le corps du barrage, dans sa fondation ou au contact;

- la réalisation d'essais mécaniques en forages, de type pressomètre ou dilatomètre:

- l'équipement ultérieur de certains sondages en piézomètres à tube ouvert ou la pose de capteurs de pression interstitielle à divers niveaux (on peut envisager jusqu'à trois capteurs par sondage).

Quelques sondages carottés sont donc indispensables dès que l'on souhaite réaliser un diagnostic approfondi d'un barrage en maçonnerie. Ils permettent d'évaluer, bien que de façon ponctuelle les caractéristiques de base entrant dans la vérification de la stabilité de l'ouvage :

- densité de la maçonnerie;

- niveau exact de la fondation;

- nature du contact:

- valeur des sous-pressions,...

Toutefois, les sondages carottés sont d'un coût élevé, ce qui en limite le nombre, en particulier pour des propriétaires d'ouvrages au départ peu motivés par les problèmes de sécurité. La richesse des informations que 
fournissent les sondages trouve ainsi ses limites dans leur représentativité, et une reconnaissance basée sur ces seules investigations ponctuelles ne permet pas de déceler d'éventuelles zones particulièrement médiocres situées à distance des forages.

De ce point de vue, la reconnaissance sismique apporte à moindre coût un complément d'information très utile. Les différentes techniques décrites ci-après offrent un large choix d'investigations, car on peut opérer soit entre sondages (profils de rive à rive), soit entre sondages et parements, soit entre parements amont et aval, que le barrage soit vide ou plein. Le programme des recon. naissances sismiques peut ainsi être adapté au mieux à chaque cas particulier d'ouvrage.

\section{LES MÉTHODES DE TRANSPARENCE SISMIQUE}

\subsection{Principe général de la transparence sismique}

Les reconnaissances sismiques par transparence servent à mesurer des vitesses sismiques au sein d'un ouvrage massif. Comme pour la sismique réfraction de surface, le principe est d'émettre un choc et de mesurer le temps de propagation de l'onde de choc, puis d'en déduire la vitesse sismique du ou des matériaux traversés. En sismique de surface, on enregistre pour un même choc un grand nombre de trajets, soit un trajet direct pour le matériau de surface et plusieurs trajets réfractés sur des matériaux plus profonds. Pour un ouvrage massif (barrage-poids par exemple), la présence de parements extérieurs ou d'orifices tels que forages ou galeries permet de choisir des couples émission-réception pour lesquels les trajets des ondes de choc sont bien connus. Cela élimine un grand nombre d'inconnues et rend le dépouillement plus aisé. On pourra alors profiter de cette simplification, soit pour élargir la zone investiguée, soit pour enrichir l'investigation en obtenant une vérjtable carte sismique.

Comme en sismique de surface, on peut se contenter d'enregistrer les ondes de compression, ou ondes $P$ mais on peut aussi enregistrer les ondes de cisaillement ou ondes S. Les ondes S étant plus lentes sont a priori difficiles à visualiser au sein d'une vibration complexe. Leur mesure nécessite donc un matériel plus performant tant pour l'émission que pour la réception.

La connaissance conjointe des vitesses des ondes $\mathrm{S}$ et des ondes $\mathrm{P}$ permet de calculer les modules dynamiques du matériau. C'est d'ailleurs essentiellement la vitesse des ondes $\mathrm{S}$ qui intervient dans ces calculs.

Il est donc essentiel de savoir avant de choisir un type d'investigation si l'on veut seulement mesurer les vitesses sismiques dans le corps de l'ouvrage ou bien si l'on veut aussi connaître les modules dynamiques.

\subsection{Les dispositifs d'émission}

Pour émettre un signal sonore (ou une vibration) on utilise le plus souvent:

- des explosifs;

- des étinceleurs ;

- une source à frappe;

- un canon à air.

- L'explosif est un moyen pratique et peu encombrant. Il doit être utilisé dans un forage maintenu en eau. Lorsqu'on désire produire un signal perçu à longue distance, cette technique peut dégrader le forage. $\mathrm{Ce}$ type de source produit essentiellement des efforts de compression (ondes P).

- L'étinceleur délivre une onde de choc qui est créée par l'application d'une forte différence de potentiel entre deux électrodes. Il délivre un signal plus propre mais moins énergétique que l'explosif. Il est réalisé dans un forage en eau et produit essentiellement des ondes P. Son principe de fonctionnement (forte ddp) nécessite des précautions particulières d'utilisation.

- Le principe de la source à frappe est de transformer un choc vertical, produit par exemple par un marteau, en effort de cisaillement autour du forage grâce à un système pneumatique ou autre parfaitement plaqué à la paroi du forage. Il n'est pas indispensable que le forage soit en eau. L'énergie délivrée ainsi permet de générer aussi bien des ondes $\mathrm{P}$ que des ondes $\mathrm{S}$.

- Enfin, le canon à air (fig. 1) a pour principe la libé. ration brutale, sous l'eau, d'air fortement comprimé. Cette source assez énergétique, d'une portée de plusieurs dizaines de mètres est difficilement utilisable en forage. En revanche, elle est bien adaptée à l'émission de signaux contre la partie immergée du parement amont d'un barrage. Le canon à air génère essentiellement des ondes $\mathrm{P}$.

En résumé, l'explosif, l'étinceleur et le canon à air sont bien adaptés pour générer des ondes P. L'utilisation de
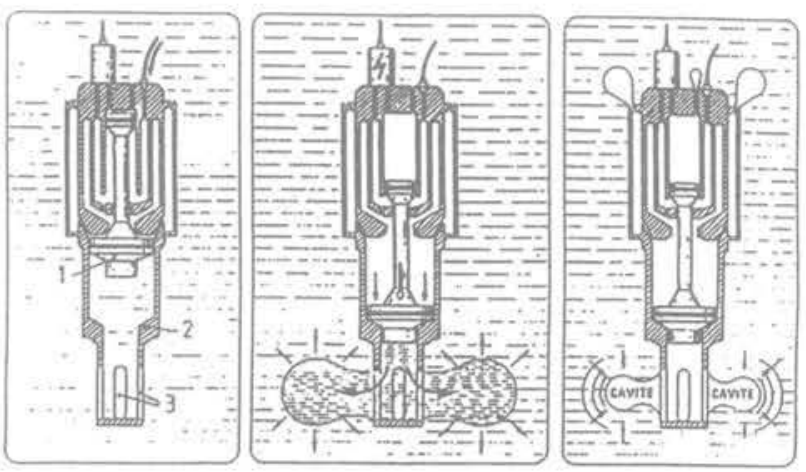

Fig. 1. - Principe de fonctionnement du canon à air.

A: Avant le tir; $B$ : Après le tir; $C$ : Réarmement. 1: Piston : 2: Butée; 3 : Events.

Fig. 1. - Air-gun description

A: Before the shot: $B$ : After the shot: $C$ : Rearmed. 1: Suttle: 2: Thrust: 3 : Orifices. 
l'explosif en forage est limitée du fait des risques de dégradation du forage dès que l'on travaille sur des portées longues. Pour générer des ondes $\mathrm{S}$ de bonne qualité, les sources à frappe sont de loin les mieux adaptées.

\subsection{Les dispositifs de réception et d'enregistrement}

Les dispositifs de réception sont soit des hydrophones, soit des géophones. Les premiers ne fonctionnent que dans l'eau et les seconds fonctionnent aussi bien dans l'eau qu'hors d'eau.

\subsubsection{Les hydrophones}

Les hydrophones enregistrent, par des capteurs piézoélectriques, les variations de pression de l'eau dans laquelle ils se trouvent. Ils ne captent correctement que les ondes de compression (ondes P). En contrepartie, ils présentent l'avantage d'être peu encombrants et, reliés à un câble sismique, ils peuvent être mis en place simultanément (au nombre de 12 ou 24) dans un forage pour réceptionner un même impact. L'utilisation de ce câble à hydrophones sera décrite plus loin pour la technique du carottage sismique $(\$ 2.4 .1)$ et pour la tomographie (\$2.4.5).

\subsubsection{Les géophones}

Les géophones enregistrent les vitesses particulaires au moyen de bobines dont le déplacement engendre un signal électrique. Ils permettent de capter aussi bien les ondes $\mathrm{P}$ que les ondes $\mathrm{S}$. Pour fournir des enregistrements de bonne qualité, les géophones doivent être parfaitement plaqués au terrain. Lorsqu'on les utilise en forage, le couplage correct géophone-terrain se fait au moyen d'un dispositif de type pneumatique, relativement lourd et encombrant. De ce fait, alors que le nombre de points de réceptions simultanées est de 12 ou 24 lorsqu'on utilise des hydrophones, il n'est que de 1 ou 2 lorsque la réception se fait par géophones plaqués à la paroi. Ceci est pénalisant en particulier pour les tomographies qui nécessitent de nombreux couples émission-réception.

\subsubsection{Les sondes formées de trois géophones.}

Pour réceptionner à la fois des ondes $\mathrm{P}$ et des ondes $\mathrm{S}$, on utilise une sonde comportant trois géophones $(1 \mathrm{~V}+2 \mathrm{H})$ vibrant dans trois directions orthogonales. Pour que les ondes S réceptionnées soient claires, il est également nécessaire que ces sondes soient soigneusement plaquées aux parois du forage ou scellées sur les parements de l'ouvrage.

\subsubsection{La chaîne de mesure}

Les laboratoires sismiques modernes sont tous des laboratoires numériques qui permettent la sommation des signaux. Cette propriété est essentielle pour améliorer le rapport signal/bruit. Ils présentent très généralement des caractéristiques satisfaisantes pour l'ensemble des essais décrits ci-après: gain suffisant, toutes possibilités de filtres, gains de sortie, enregistrement sur disquettes ou bandes magnétiques pour traitements ultérieurs.

\subsection{Les différentes méthodes de transparence sismique}

Compte tenu des performances des matériels, de leurs contraintes, de la nécessité ou non d'enregistrer des ondes $\mathrm{S}$, les différents essais suivants peuvent être pratiqués. Ils sont résumés dans le tableau.

\subsubsection{Le carottage sismique}

Le carottage sismique est l'essai le plus simple. Il utilise un seul forage. Une grappe d'hydrophones ou de géophones moulés sur un câble est descendue dans le forage (fig. 2). On peut ainsi enregistrer 12 ou 24 signaux à différentes profondeurs, pour une même émission de surface. L'essai permet donc l'enregistrement des vitesses des ondes $\mathrm{P}$ en fonction de la profondeur. Il est nécessaire de maintenir le forage en eau pour assurer le couplage récepteur-terrain.

Cette méthode rapide valorise les sondages et en particulier les sondages destructifs. Elle complète des méthodes sismiques plus lourdes, telles que la tomographie (voir $\$ 2.4 .5$ ).

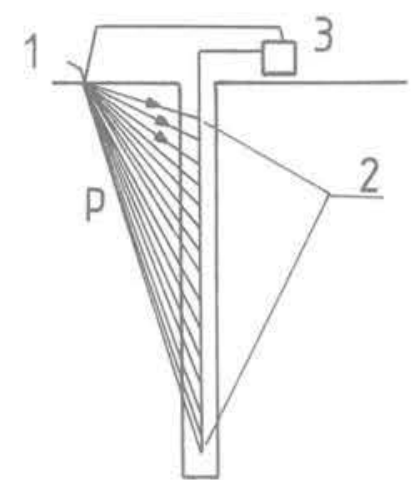

Fig. 2, - Le carottage sismique.

1: Emission tir; 2: Réception; 3: Laboratoire.

Fig. 2. - Seismic logging.

1: Shot point: 2: Reception: 3: Seismograph.

\subsubsection{L'essai cross-hole}

L'essai cross-hole est réalisé entre deux forages, l'un consacré à l'émission et l'autre à la réception (fig. 3). Classiquement, cet essai destiné à valoriser au mieux les sondages est utilisé pour mesurer les vitesses des ondes $\mathrm{P}$ et des ondes $\mathrm{S}$, sur des trajectoires horizontales. Les ondes de cisaillement sont des ondes $\mathrm{S}_{\mathrm{v}} \mathrm{vi}-$ brant dans le sens vertical.

La difficulté d'isoler une onde tardive $\mathrm{S}$ au milieu d'une vibration complexe amène:

- à étudier les seuls trajets horizontaux en plaçant émetteur et récepteur au même niveau;

- à utiliser une source qui produit un effort de cisail. lement énergétique ;

- à utiliser un capteur capable de bien réceptionner des ondes S. 
Tableau - Les différentes techniques de transparence sismique pour reconnaître un barrage.

\begin{tabular}{|c|c|c|c|c|c|}
\hline $\begin{array}{l}\text { Dénomination } \\
\text { de l'essai }\end{array}$ & $\begin{array}{c}\text { Implantation } \\
\text { des investigations }\end{array}$ & Emission & Réception & Trajets étudiés & Paramètres mesurés \\
\hline $\begin{array}{l}\text { Carottage } \\
\text { sismique }\end{array}$ & $\begin{array}{l}1 \text { forage } \\
\text { (en eau) }\end{array}$ & Tir en surface & $\begin{array}{l}\text { Grappe de } n \text { géophones } \\
\text { ou d'hydrophones }\end{array}$ & $\begin{array}{l}n \text { trajets } \\
\text { sensiblement } \\
\text { verticaux }\end{array}$ & $\begin{array}{l}\text { Ondes } P \\
\text { (vitesses verticales) }\end{array}$ \\
\hline Cross-hole & 2 forages & $\begin{array}{l}\text { Source à frappe } \\
\text { déplacée en } n \text { points }\end{array}$ & $\begin{array}{l}\text { Sonde formée } \\
\text { de } 3 \text { géophones } \\
(1 \mathrm{~V}+2 \mathrm{H} \text { ) déplacée } \\
\text { aux mêmes niveaux }\end{array}$ & $\begin{array}{l}n \text { trajets } \\
\text { horizontaux }\end{array}$ & $\begin{array}{l}\text { Ondes } \mathrm{P} \text { et } \mathrm{S}_{\mathrm{v}} \\
\text { (vitesses } \\
\text { horizontales) }\end{array}$ \\
\hline \multirow[t]{2}{*}{ Down-hole } & 2 forages & $\begin{array}{l}\text { Source à frappe } \\
\text { à l'une des extrémités } \\
\text { d'un forage }\end{array}$ & \multirow{2}{*}{$\begin{array}{l}\text { Sonde formée } \\
\text { de } 3 \text { géophones déplacée } \\
\text { à diverses profondeurs }\end{array}$} & \multirow[t]{2}{*}{$\begin{array}{l}\text { Trajets } \\
\text { sensiblement } \\
\text { verticaux }\end{array}$} & $\begin{array}{l}\text { Ondes } P \text { et } S_{v} \\
\text { (vitesses verticales) }\end{array}$ \\
\hline & 1 forage & $\begin{array}{l}\text { Dispositif de frappe } \\
\text { transversal en surface }\end{array}$ & & & $\begin{array}{l}\text { Ondes } P \text { et } S_{h} \\
\text { (vitesses verticales) }\end{array}$ \\
\hline \multirow[t]{2}{*}{$\begin{array}{l}\text { Transparence } \\
\text { simple }\end{array}$} & 2 parements & \multirow{2}{*}{$\begin{array}{l}\text { Canon à air dans le cas } \\
\text { d'un parement en eau } \\
\text { Frappe dans le cas } \\
\text { d'un parement } \\
\text { hors d'eau }\end{array}$} & \multirow{2}{*}{$\begin{array}{l}\text { Géophones scellés } \\
\text { au parement aval } \\
\text { Géophone ou hydrophone } \\
\text { dans le forage }\end{array}$} & \multirow[t]{2}{*}{$\begin{array}{l}1 \text { trajet ou } n \\
\text { trajets étudiés } \\
\text { indépendamment }\end{array}$} & \multirow[t]{2}{*}{ Ondes P } \\
\hline & $\begin{array}{l}1 \text { forage } \\
\text { et } 1 \text { parement }\end{array}$ & & & & \\
\hline \multirow[t]{2}{*}{ Tomographie } & \multirow{2}{*}{$\begin{array}{l}2 \text { forages } \\
\text { ou parement } \\
\text { et forage } \\
\text { ou } 2 \text { parements }\end{array}$} & \multirow{2}{*}{$\begin{array}{l}\text { Source à frappe } \\
\text { déplacée en } n \text { points ou } \\
\text { explosifs peu puissants } \\
\text { placés en n points }\end{array}$} & $\begin{array}{l}\text { Grappe de } p \text { hydrophones } \\
\text { si forage en eau }\end{array}$ & \multirow[t]{2}{*}{$\begin{array}{l}\mathrm{n} \times \mathrm{p} \text { trajets } \\
\text { quelconques }\end{array}$} & \multirow[t]{2}{*}{$\begin{array}{l}\text { Ondes P } \\
\text { (éventuellement } \\
\text { ondes S) }\end{array}$} \\
\hline & & & $\begin{array}{l}\text { Sonde de } 3 \text { géophones } \\
\text { déplacés en p points }\end{array}$ & & \\
\hline
\end{tabular}

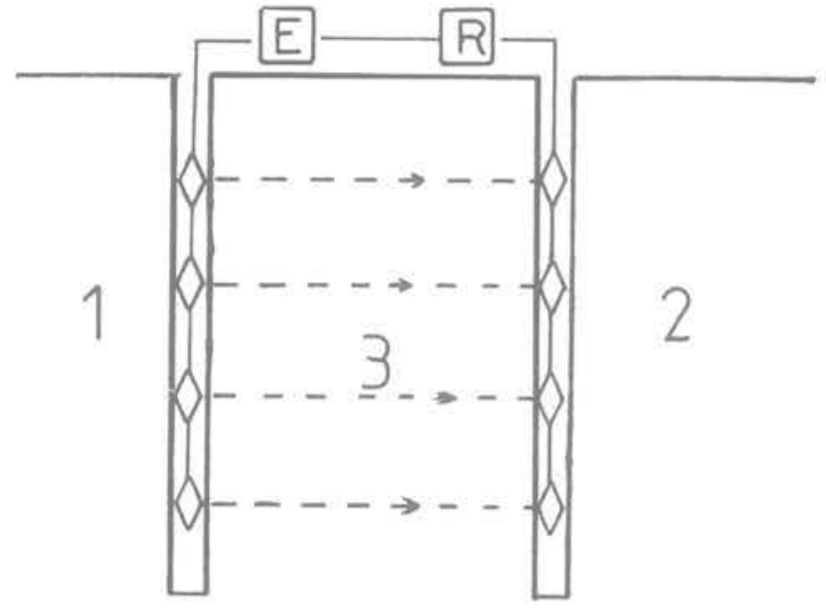

Fig. 3. - L'essai cross-hole.

1: némissions : 2 : n réceptions par dispositif plaqué à la paroi; 3 : $n$ trajets horizontaux.

Fig. 3. - Cross-hole test.

$1:$ nemissions ; $2:$ n receptions (reception device against the hole). 3 : n horizontal raypaths.

On utilise de préférence une source à frappe plaquée contre la paroi du forage, déplacée successivement en chaque point d'émission. Cependant, la spécificité de la sonde qui crée du cisaillement n'est pas de nature à permettre de grandes portées et limite donc la distance entre sondages. La sonde de réception, composée de trois géophones, est plaquée également aux parois et déplacée aux mêmes niveaux.

Le dépouillement classique de l'essai cross-hole suppose des forages distants de 5 à $10 \mathrm{~m}$. Si les forages sont plus éloignés, jusqu'à 20 ou $30 \mathrm{~m}$, il est nécessaire de considérer que les trajets les plus courts en temps ne sont pas obligatoirement les trajets directs, mais des trajets éventuellement réfractés sur des horizons plus rigides. On doit tenir compte de l'ensemble des mesures à différents niveaux et établir un modèle de succession de vitesses cohérent. Ceci nécessite un programme de traitement automatique des temps d'arrivée. Le calcul doit tenir compte de la courbure des rayons (vitesses variant progressivement avec la profondeur) et/ou de leur réfraction (horizons bien caractérisés en couches).

Les signaux peuvent être traités en analyse de fréquence. Avec les sondes à frappe, les fréquences des ondes $\mathrm{P}$ sont de l'ordre de 1000 à 1400 Hertz et celles des ondes S de 150 à 300 Hertz. Lorsque la source est bien adaptée, cette reconnaissance des signaux par leur fréquence est suffisamment caractéristique pour qu'aucun traitement ne soit nécessaire. Avec les sondes tridirectionnelles, la distinction entre ondes $\mathrm{P}$ et ondes S est facilitée, car leurs arrivées respectives sont enregistrées par des capteurs différents (fig. 7).

\subsubsection{L'essai down-hole}

L'essai down-hole est dérivé de l'essai cross-hole. Comme lui, il mesure les vitesses des ondes $\mathrm{P}$ et $\mathrm{S}$, mais sur des trajectoires d'ondes verticales et non ho- 
rizontales. Il existe deux variantes de cet essai. Dans la première, l'émission est placée près d'une extrémité d'un forage et les réceptions près de l'autre extrérnité du second forage, assez rapproché du premier (fig. 4a). On génère alors des ondes $\mathrm{P}$ et des ondes $\mathrm{S}_{v}$ (ondes de cisaillement dans la direction verticale)

La seconde variante n'utilise qu'un seul forage en disposant d'une source de frappe transversale au sol et placée en surface (fig. 4b). On génère alors des ondes $P$ et des ondes $S_{h}$ (ondes de cisaillement dans la direction horizontale). Comparé à l'essai cross-hole, cet essai qui utilise les mêmes matériels, permet de lever des indé. terminations dans le cas d'hêtérogénéités dans le sens horizontal. L'essai cross-hole suppose en effet que les couches traversées sont homogènes dans le sens horizontal. L'essai dow-hole est plus fréquemment utilisé pour les études de fondation de centrales nucléaires.

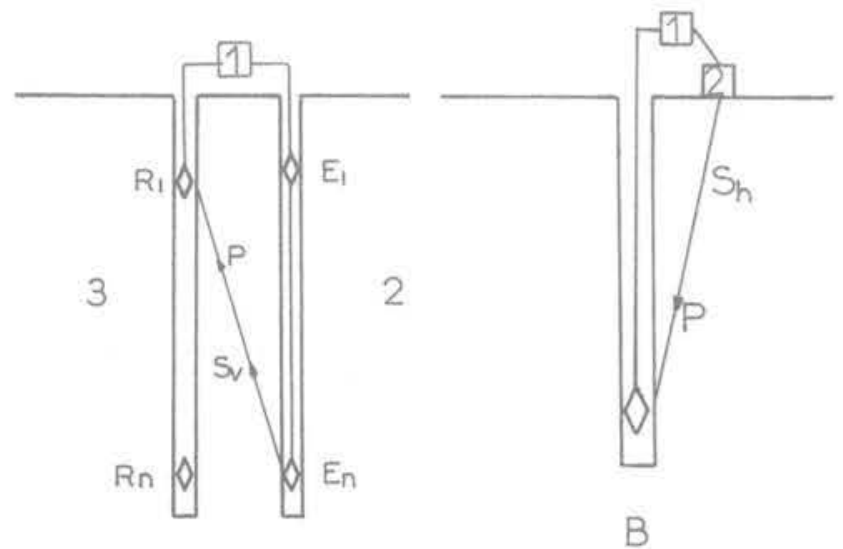

A

Fig. 4. - L'essai down-hole.

1: Laboratoire; 2: Emission (E1 et E2) ; 3 : Réception (R1 à $R n$ ).

Fig. 4. - Down hole test.

1: Seismograph: 2: Shot point (E1 and E2). 3: Reception (R1 to Rn).

\subsubsection{La transparence simple}

En variante de l'essai de base cross-hole réalisé entre deux forages, il s'avère possible d'utiliser un parement ou les deux pour placer les émetteurs et les récepteurs. Dans le cas d'un barrage on peut donc:

- placer les émetteurs sur le parement aval, et les récepteurs dans un forage;

- placer les émetteurs sur le parement amont et les récepteurs dans un forage ou sur le parement aval (fig. 5).

L'essai de transparence simple étudie donc un barrage plutôt dans le sens amont-aval par opposition à l'essai cross-hole qui l'étudie en général dans le sens rive à rive. L'intérêt le plus net de cette technique est d'être

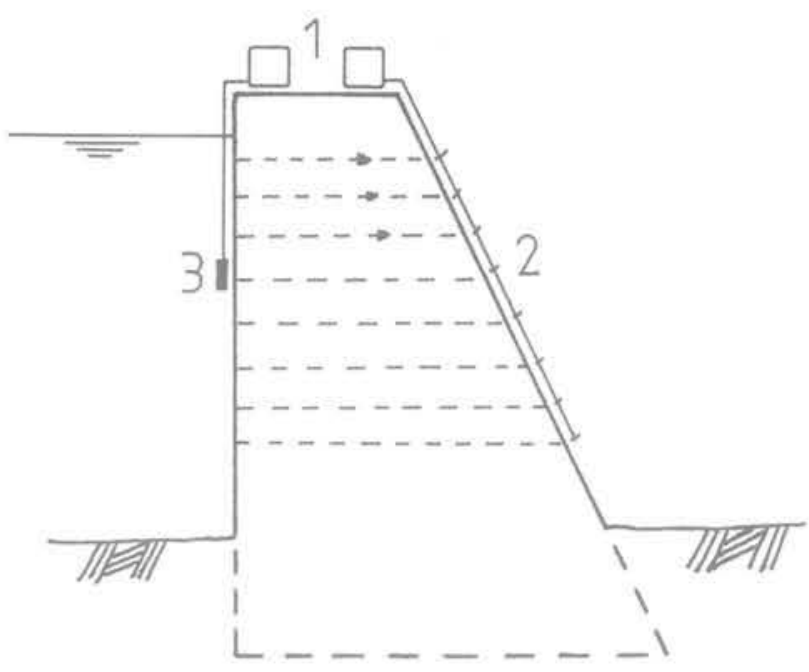

Fig. 5. - La transparence sismique.

1: Laboratoire; 2: Géophones; 3 : Canon à air.

Fig. 5. - Direct seismic measures.

1: Seismograph: 2: Geophones: 3: Air gun

applicable en l'absence de forage. En contrepartie, il faut dans ce cas plaquer les sondes de réception sur le parement aval, ce qui exige des échaffaudages ou le recours à des alpinistes. Toujours dans le cas où l'on travaille sans forages, la technique du canon à air permet de délivrer des ondes de qualité depuis un parement amont en eau, mais ne permet de bien mesurer que des ondes $\mathrm{P}$.

\subsubsection{La tomographie}

On appelle tomographie toute méthode de détermination des vitesses sismiques grâce au balayage d'un plan d'expérimentation (ou panneau) par des rayons sismiques et à une interprétation des mesures par un processus d'inversion automatique.

Sur une ligne on dispose p récepteurs qui restent fixes pendant toute l'expérimentation. Sur une autre ligne on déplace l'émetteur successivement en $n$ points. Pour chaque point d'émission on mesure les temps d'arrivée aux p récepteurs (fig. 6). On dispose ainsi de n.p informations que l'on traite à l'aide de programmes automatiques d'inversion permettant d'établir une carte des vitesses sismiques.

Pour une reconnaissance de barrage, la tomographie peut se faire entre deux forages, entre un forage et un parement ou entre le parement amont et le parement aval.

L'interprétation des mesures suppose que deux rayons sismiques voisins suivent par continuité des trajets voisins mais pas nécessairement droits.

Cet essai est en principe réservé à l'étude des ondes $\mathrm{P}$ seulement, car la mesure des temps d'arrivée des 


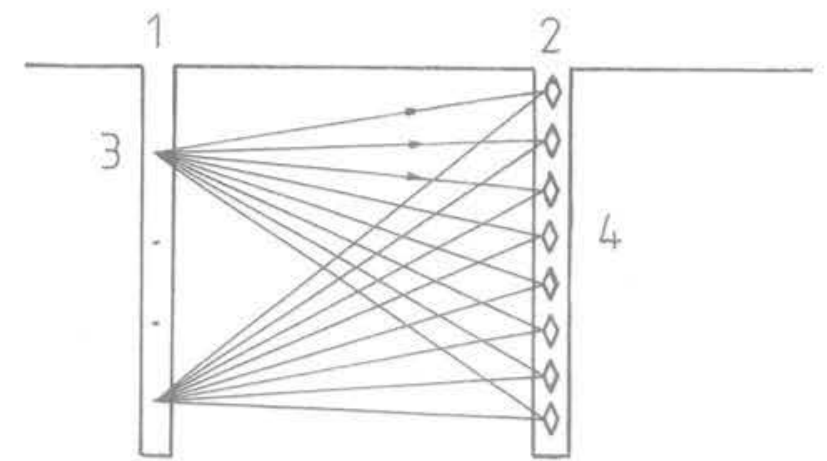

Fig. 6. - La tomographie: analyse en ondes $P$ de n.p rayons et traitement automatique.

1: Forage d'émission; 2 : Forage de réception. 3: $n$ émissions; 4 : p réceptions.

Fig. 6. - Tomography: $P$ waves analysis of $n, p$ rays and automatic treatment

1: Transmitting drilt hole: 2 : Receiving dill hole. $3:$ in shots: $4:$ : p receptions.

ondes S n'a pas, pour l'instant, une précision compatible avec l'exigence du processus d'inversion automatique.

Les émissions peuvent être pratiquées à l'explosif à condition de ne pas dégrader le forage qui doit être utilisé de nombreuses fois, d'où tout l'intérêt des sources à frappe qui dégradent moins le forage à énergie donnée. La figure 9 montre l'exemple d'une carte des vitesses obtenues entre les trois forages de reconnaissance pratiqués au barrage de la Gimond, distants de $15 \mathrm{~m}$ et $25 \mathrm{~m}$.

\section{DEUX RECONNAISSANCES PILOTES DE BARRAGES EN MAÇONNERIE}

Dans le cadre de l'étude de diagnostic de sécurité de barrages anciens, le CEMAGREF a eu l'occasion de proposer une méthodologie de reconnaissance basée sur des sondages carottés et sur la valorisation de ces sondages par des études géophysiques. Les deux campagnes géophysiques que nous allons décrire ont été pilotées par le CEMAGREF et réalisées par SEGG.

\subsection{Le barrage de la Bourne (1980)}

Le barrage de la Bourne est un barrage poids déversant en maçonnerie destiné à alimenter un canal d'irrigation. Construit en 1870 , il est haut de $18 \mathrm{~m}$ sur la molasse de fondation. Le parement amont est vertical et le parement aval a un fruit de 0,57 , a priori largernent insuffisant.

\subsubsection{Le programme des reconnaissances}

Le diagnostic du barrage a été réalisé en 1980. Les reconnaissances ont comporté la réalisation depuis la crête de quatre sondages carottés descendus de $5 \mathrm{~m}$ dans la fondation. L'hétérogénéité des carottes a conduit le CEMAGREF à lancer une campagne de reconnaissance géophysique par méthodes micro-sismiques et micro-électriques.

Les mesures sismiques ont comporté (fig. 7):

- des carottages sismiques avec émission en tête de chaque forage et réception tous les $50 \mathrm{~cm}$;

- des mesures par transparence simple avec émission du choc au pied aval du barrage et réception par des géophones placés dans les sondages tous les mètres.

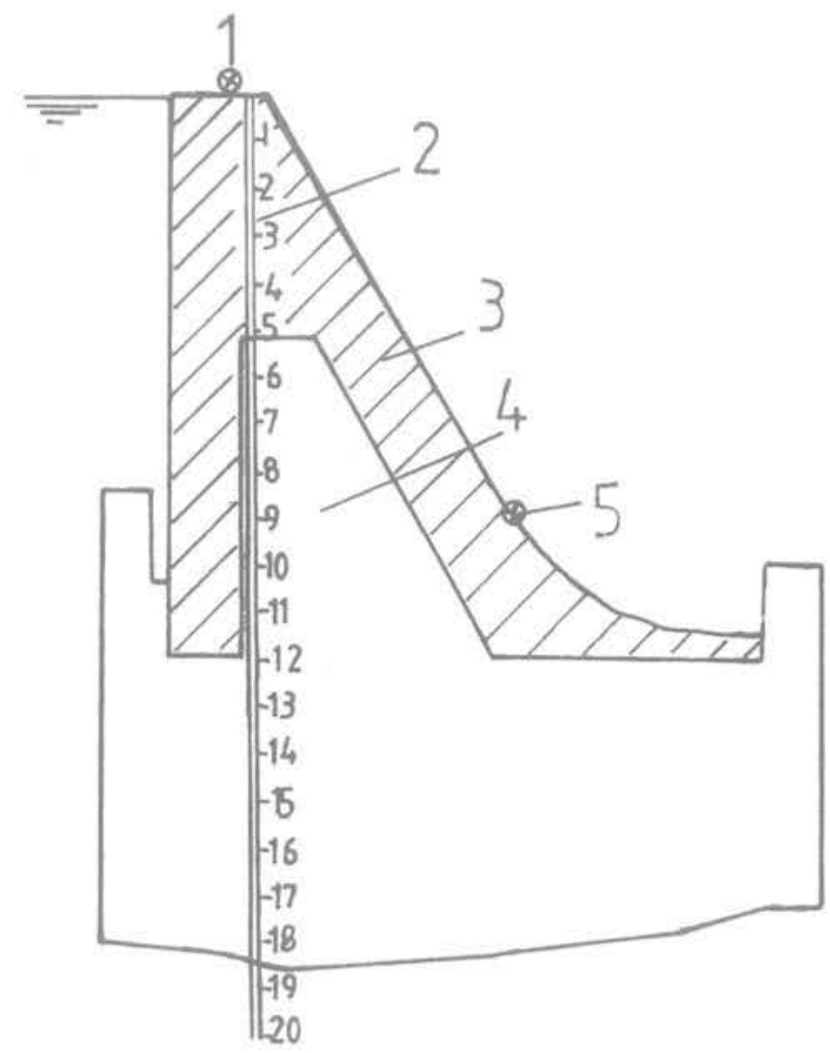

Fig. 7. - Barrage de la Bourne - Vue en coupe en partie centrale.

1: Ebranlement pour carottages sismiques ; 2 : Sondage de réception; 3: Maçonnerie; 4: Béton maigre; 5: Ebranlement par transparence.

Fig. 7. La Bourne dam cross section in central part.

1. Shot point for seismic logging: 2: Reception hole:

3: Masonry: 4: Lean concrete:

5: Shot point for direct measures.

Il est à noter que les méthodes employées utilisaient seulement les sondages un par un, contrairement aux méthodes de type cross-hole. En outre, les mesures par transparence simple se sont appuyées sur les quatre sondages, parce qu'il n'était pas économiquement envisageable de vider la retenue, et que les techniques d'émission puissante sous l'eau n'étaient pas encore classiquement utilisées.

Par ailleurs, des mesures micro-électriques ont été réalisées avec une électrode d'émission implantée en rive 
à une distance supposée infinie, une autre électrode d'émission placée dans le forage et deux électrodes de réception dans le forage également. Le forage était maintenu en eau à l'aide d'une pompe.

\subsubsection{Les résultats}

Les sondages ont montré que le corps de l'ouvrage était relativement poreux avec une perméabilité extrapolée de 25 UL alors que les parements et la crête montraient des perméabilités de 1 à 3 UL. Le matériau carotté s'est révélé de qualité hétérogène parfois très bon, parfois poreux ou caverneux. L'un des quatre sondages a dû être tubé en partie basse ; il s'agit de celui qui intéresse la zone où le béton de fondation avait été mis en place sous l'eau.

Les résultats de la campagne géophysique ont permis de bien distinguer:

- la maçonnerie formant carapace avec une résistivité de $180 \Omega \times \mathrm{m}$ et une vitesse sismique de $2300 \mathrm{~m} / \mathrm{s}$;

- le béton du coeur du barrage avec une résistivité plus faible de $130 \Omega \times \mathrm{m}$ et une vitesse sismique de l'ordre de $4000 \mathrm{~m} / \mathrm{s}$;

- le béton de fondation avec une résistivité de $140 \Omega \times$ m et une vitesse de $4100 \mathrm{~m} / \mathrm{s}$;

- la molasse de fondation avec une résistivité de $80 \Omega \times \mathrm{m}$ et une vitesse de $2500 \mathrm{~m} / \mathrm{s}$.

On a pu associer les faibles valeurs de la résistivité à la présence de béton poreux.

La campagne a, en outre, montré que les carottes obtenues par le sondage qui avait dû être tubé, donnaient une vision trop pessimiste de la qualité réelle du béton (un caillou entraîné par la couronne ayant pu désagréger les matériaux forés). Il a enfin été confirmé que les sondages n'étaient pas passés à côté de zones de qualité médiocre. Une reconnaissance limitée à des sondages carottés écartés de $15 \mathrm{~m}$ a donc pu être validée par un complément peu coûteux de géophysique (DEGOUTTE, 1986).

Une opération similaire a également été réalisée avec succès en 1986 au barrage de Moulin-Bertrand sur l'Hérault construit en 1922 et haut de $13 \mathrm{~m}$.

\subsection{Le barrage de la Gimond (1991)}

\subsubsection{Cadre de l'intervention}

Il s'agit d'un barrage-poids en maçonnerie servant à l'alimentation en eau potable. Construit en 1923-1925, il a été rehaussé d'environ $2 \mathrm{~m}$ en 1957 , ce qui porte sa hauteur maximum à $19 \mathrm{~m}$ sur fondation. Un remblai de $5 \mathrm{~m}$ vient l'épauler à l'aval.

Lors d'une première visite de l'ouvrage en 1989 le CEMAGREF a mis en évidence les points suivants :

- sous-dimensionnement notoire de l'évacuateur de crues;

- présence d'un abondant placage de calcite sur la partie visible du parement aval, masquant partiellement les fuites et indice d'une dissolution du liant de la maçonnerie (chaux hydraulique) ;

- quelques fuites observables ne représentant proba. blement qu'une faible part des percolations à travers l'ouvrage, compte tenu du remblai aval.

Par ailleurs, quelques calculs simples de stabilité ont montré que, depuis le rehaussement, la sécurité du barrage était limite, en particulier en cas de forte crue. Cela a conduit à convaincre le maître d'ouvrage d'engager un programme de reconnaissances approfondies qui, outre les aspects hydrologie et hydraulique de l'évacuateur, a porté essentiellement sur la structure de l'ouvrage. Cinq sondages carottés ont été réalisés, trois verticaux depuis le couronnement (S1, S2 et S3) et deux inclinés depuis le pied aval (S4 et S5), avec essais de laboratoire, essais de perméabilité de type Lugeon, essais sismiques et équipement en piézomètres.

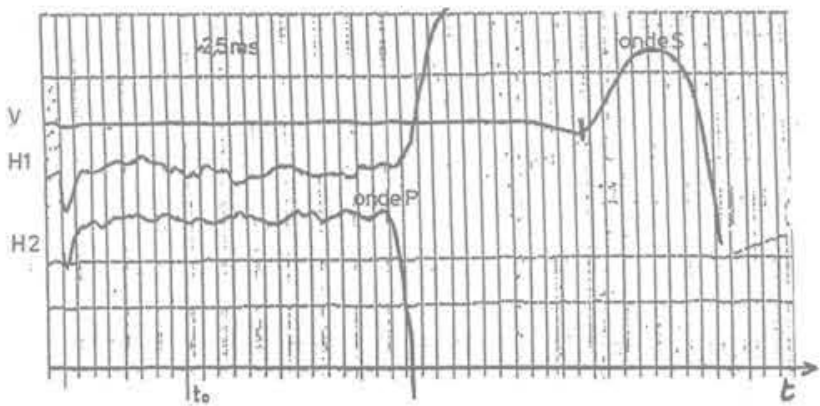

Fig. 8. - Exemple d'enregistrement fourni par une sonde composée de 3 géophones $(1 \mathrm{~V}$ et $2 \mathrm{H})$.

Barrage de la Gimond, S2-S1, $9 \mathrm{~m}$ de profondeur.

$V$ : onde $S ; H 1, H 2$ : ondes $P$.

Fig. 8. - Recording with a 3 geophones device $(1 \mathrm{~V}$ and $2 \mathrm{H})$ Gimond dam, S2-S1, $9 \mathrm{~m}$ depth.

V: Swave: H1, H2: P waves.

\subsubsection{Reconnaissance sismique}

Le but de cette auscultation était de réaliser un zonage des vitesses sismiques pour vérifier l'homogénéité de la maçonnerie ou, au contraire, pour localiser les zones où le corps de l'ouvrage était plus dégradé que ne le montraient les carottes. Contrairement au barrage de la Bourne, les techniques utilisées ont permis d'étudier à la fois des plans amont-aval et des plans rive à rive. Cette approche tridimensionnelle permet une bonne connaissance de l'ensemble de la structure. Une zone de maçonnerie particulièrement mauvaise dans le sondage central, a priori destiné à être le pivot des mesures cross-hole et de la tomographie a entrainé des difficultés d'équipement de ce sondage, le rendant impropre aux mesures sismiques. De ce fait l'émission au droit de ce forage a été faite dans la retenue sur une ligne verticale le long du parement amont au moyen d'un canon à air. Le décalage de $2,20 \mathrm{~m}$ qui s'en suit, n'entraîne pas de modification significative dans l'interprétation des mesures.

La campagne de reconnaissance sismique a comporté : - deux séries d'essais cross-hole entre les trois sondages de crête avec enregistrement des ondes $\mathrm{P}$ et ondes S; 
- un double panneau vertical de tomographie entre les trois sondages de crête portant sur les ondes $\mathrm{P}$; - trois plans subhorizontaux de transparence simple entre parements en ondes $P$;

- des carottages sismiques dans deux sondages de crête en ondes S.

\subsubsection{Résultats de la campagne sismique}

a. Cross-hole

Les figures 10 et 11 fournissent les résultats des essais cross-hole entre d'une part S2 et S3 et d'autre part S2 et $\mathrm{S} 1$. Les vitesses mesurées des ondes $\mathrm{P}$ et des ondes $\mathrm{S}$ permettent de déduire :

- le module d'Young dynamique:

- valeur moyenne : $22400 \mathrm{MPa}$,

- valeurs extrêmes : $15900 \mathrm{MPa}$ et $31300 \mathrm{MPa}$;

- le module de cisaillement dynamique:

- valeur moyenne: $8500 \mathrm{MPa}$,

- valeurs extrêmes: $6000 \mathrm{MPa}$ et $12400 \mathrm{MPa}$;

- le coefficient de Poisson:

- valeur moyenne : 0,31 ,

- valeurs extrêmes: 0,26 et 0,35 .

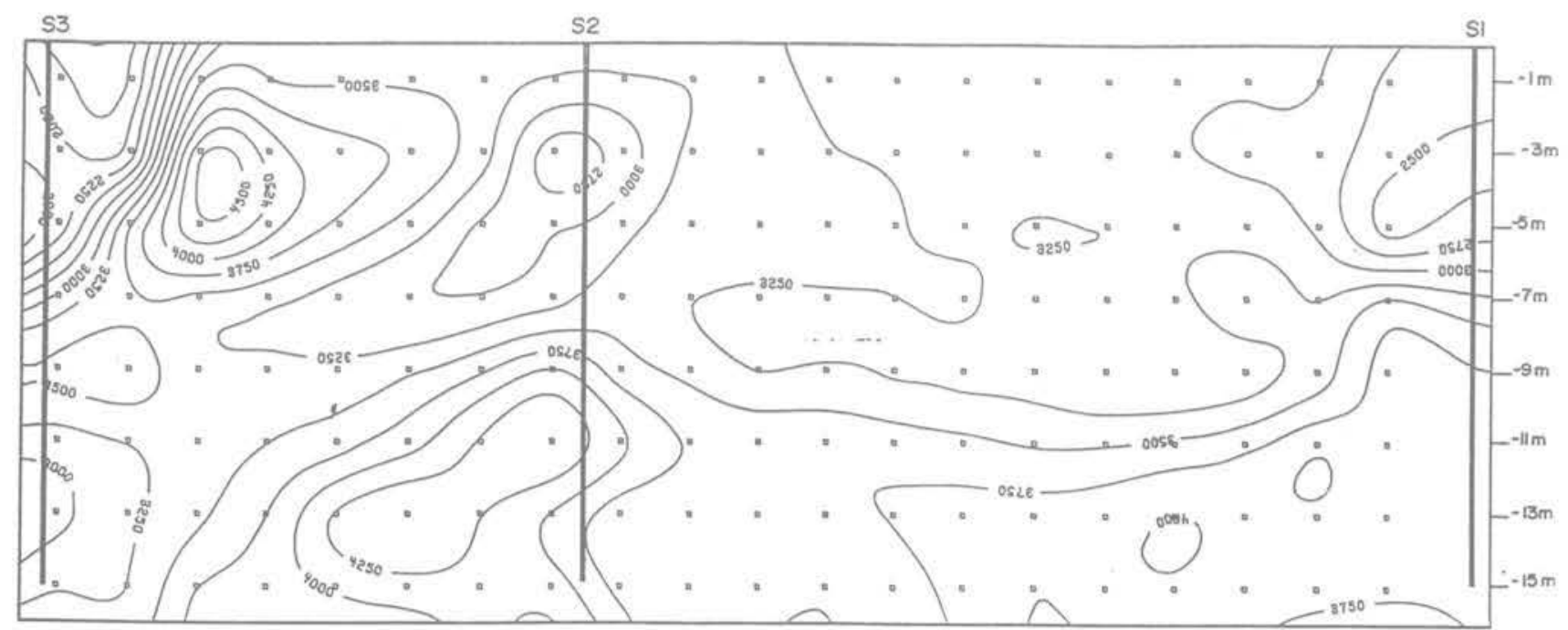

Fig. 9. - Barrage de la Gimond: carte des vitesses des ondes P, obtenue par tomographie,

Fig. 9. - Gimond Dam: map of the seismic velocities of P waves, obtained by tomography.
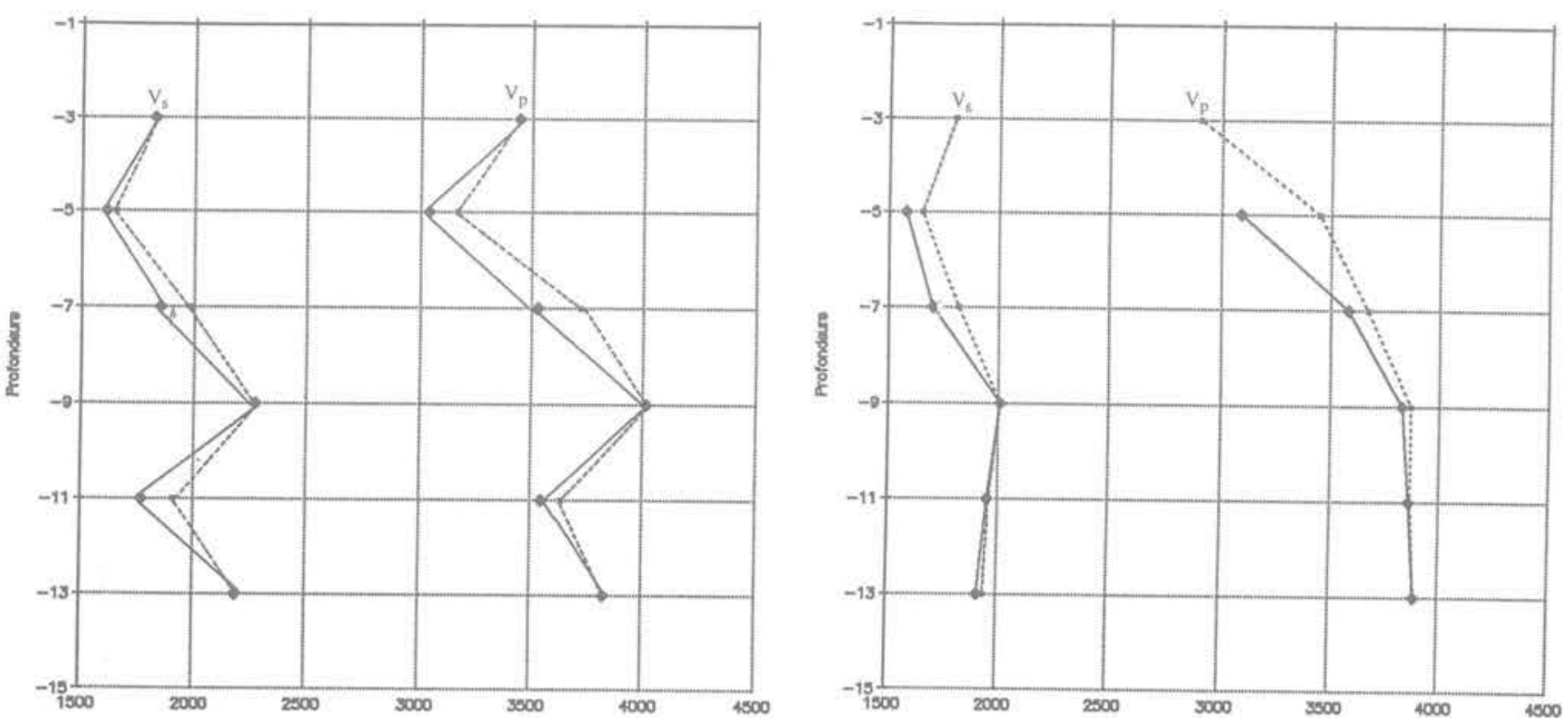

Fig. 10. et 11. - Barrage de la Gimond.

Vitesses sismiques des ondes $P$ et des ondes $S$.

Fig. 10. et 11. - Gimond Dam:

$P$ and $S$ waves seismics velocities. 
Les profils des vitesses des ondes de compression et de cisaillement montrent des zones de vitesses plus faibles, indices de maçonnerie plus médiocre de $-3 \mathrm{~m}$ à $-7 \mathrm{~m}$ sur le panneau $\mathrm{S} 2-\mathrm{S} 3$ et en partie haute du panneau S2-S1.

\section{b. Transparence simple et sismique réfraction}

Les mesures par transparence simple font apparaittre une forte anisotropie des vitesses des ondes $\mathrm{P}$ :

- vitesse moyenne de rive à rive dans le corps de l'ouvrage: $3610 \mathrm{~m} / \mathrm{s}$;

- vitesse moyenne de rive à rive sur le parement aval : $3725 \mathrm{~m} / \mathrm{s}$;

- vitesse moyenne amont aval: $2835 \mathrm{~m} / \mathrm{s}$.

Les vitesses de rive à rive sont du même ordre de grandeur que les vitesses mesurées sur le parement aval, mais elles sont en moyenne $30 \%$ supérieures aux vitesses dans le sens amont-aval. Ceci traduit le soin particulier apporté sur ce type d'ouvrage à la réalisation des parements (maçonnerie de pierres appareillées) par rapport au remplissage interne qui contient une forte proportion de liant plus ou moins dégradé.

\section{c. Tomographie}

Les mesures réalisées entre S2 et S3 et entre S2 et S1 permettent de disposer d'une carte des vitesses sismiques des ondes $\mathrm{P}$ sur un panneau moyen vertical de $40 \mathrm{~m}$ de longueur en partie centrale de l'ouvrage (fig. 9).

Globalement on note une augmentation des vitesses avec la profondeur, avec sous le niveau- $11,0 \mathrm{~m}$ des valeurs presque toujours supérieures ou égales à $3750 \mathrm{~m} / \mathrm{s}$.

Des zones de vitesses plus faibles apparaissent près des sondages en partie haute. Les zones médiocres des forages $\mathrm{S} 1$ et $\mathrm{S} 2$ ressortent aussi bien de la tomographie que des transparences sismiques. Elles sont confirmées par l'observation des carottes.

En revanche, l'anomalie cartographique au droit du fo. rage S3 semble avoir été exagérée par le processus d'inversion automatique, car cette zone de faibles vitesses est adjacente à une zone de très fortes vitesses (« téléscopage » de zones de vitesses très différentes). Cette anomalie au droit de S3 n'est d'ailleurs pas re: pérée par les transparences.

\subsubsection{Conclusion des reconnaissances du barrage de la Gimond}

L'ensemble des reconnaissances a montré que le barrage présentait des défauts d'étanchéité liés à des phénomènes de dissolution du liant, ayant créé des passages préférentiels pour l'eau. Mais du point de vue mécanique, la maçonnerie reste globalement de bonne qualité ainsi qu'en attestent l'observation des carottes, les essais mécaniques et les valeurs relativement élevées des vitesses sismiques. Les parements de l'ouvrage, réalisés avec plus grand soin, présentent des caractéristiques sensiblement meilleures que le remplissage central. On distingue quelques zones plus médiocres situées surtout en partie haute de louvrage.

Les contraintes liées à l'exploitation de ce barrage pour la fourniture d'eau potable vont probablement orienter les travaux de confortement vers la réalisation d'un voile d'injection, traitant le corps du barrage et sa fondation. Ce traitement apparaît adapté à la nature de la perméabilité affectant l'ouvrage.

\section{ENSEIGNEMENTS TIRÉS}

Les deux reconnaissances décrites (barrages de la Bourne et de la Gimond) montrent bien les progrès réalisés en dix ans dans les méthodes de reconnaissance sismique appliquées aux barrages.

En particulier, les étinceleurs et les canons à air permettent de réaliser l'émission de signaux le long d'un parement immergé. On peut donc envisager de mener une première reconnaissance sismique rapide, sans sondages et sans vidange du plan d'eau. Par une série de profils amont aval et en ne s'intéressant qu'aux ondes $P$, on peut repérer des zones d'anomalies sismiques qui guideront l'implantation des sondages carottés ultérieurs.

Par ailleurs, on dispose maintenant d'appareillages d'émission et de chaînes de réception bien adaptés pour mesurer non seulement les ondes $\mathrm{P}$ mais aussi les ondes $\mathrm{S}$, tant en forages que sur les parements.

Lors de la campagne d'essais au barrage de la Gimond, pour les essais cross-hole les enregistrements de terrain étaient d'excellente qualité. On distinguait très bien l'arrivée de l'onde $\mathrm{P}$ (très énergétique) de celle de l'onde S (fig. 8). Les temps étaient pointés à $5.10^{-5} \mathrm{~s}$ près, ce qui compte tenu des temps de parcours donne une précision de $1 \%$. Si on tient compte par ailleurs de l'erreur de verticalité des sondages (moins de $1 \%$ ) on aboutit dans ce cas à une erreur expérimentale sur les vitesses de l'ordre de $2,5 \%$.

Une telle précision permet une interprétation de type tomographie avec des programmes de calcul à rayons courbes situés dans un même plan.

Cependant ces méthodes de reconnaissances sismiques comportent un certain nombre de contraintes et de limites:

- les essais cross-hole et les tomographies nécessitent des forages d'autant plus rapprochés les uns des autres qu'on demande une reconnaissance fine. Il y a là un premier inconvénient d'ordre économique :

- lorsqu'on réalise ces forages dans un barrage pour lequel on suppose l'existence de zones dégradées ou suspectes, on risque de se heurter à des problèmes d'exécution et d'équipement de ces forages. La réalisation des essais sismiques nécessite la pose d'un tu. bage plastique scellé au terrain;

- enfin, lors d'essais cross-hole ou de tomographies réalisés rive à rive sur un barrage-poids, il est à craindre 
que la faible épaisseur de l'ouvrage conduise à des propagations préférentielles le long des parements. C'est en particulier le cas pour des ouvrages en maçonnerie dont les parements sont constitués de pierres taillées et appareillées avec plus de soin que le remplissage in terne. Ceci conduit aussi à réduire les distances entre émission et réception.

On peut donc pour des reconnaissances futures proposer la méthodologie suivante:

- En première phase, on réalise une campagne sis. mique légère basée sur la transparence simple entre les parements amont et aval, ne nécessitant ni sondages, ni vidange de la retenue. Les signaux sont émis au canon à air. Les capteurs sont scellés sur le parement aval, ce qui exige l'intervention d'alpinistes pour les grands barrages. On peut travailler sur des profils situés soit sur des plans horizontaux (investigations par couches), soit sur des plans verticaux. On cherchera à identifier les particularités éventuelles liées aux zones des parements (sismique de surface). La densité des points d'émission et de réception dépendra du degré de précision souhaité (maille de $4 \times 4 \mathrm{~m}$ à titre indicatif). Selon la qualité des signaux enregistrés, il sera parfois possible de pointer les ondes $\mathrm{S}$ et de dresser une première cartographie sommaire des modules sismiques.

- En seconde phase, au vu des résultats de la première campagne sismique, on définira le nombre et l'implantation des sondages carottés qui constitueront la base principale de la reconnaissance: examen des carottes, essais de laboratoire (densité, porosité, résistance à la compression et au fendage, vitesse sismique...), essais in situ (dilatomètre, perméabilité) et équipement en piézomètres.

- Enfin en troisième phase, on réalisera éventuellement une deuxième campagne sismique basée sur des mesures cross-hole ou tomographies entre sondages ou entre sondages et galeries en opérant sur des bases courtes et avec pour objectifs la mesure précise des modules (ondes $\mathrm{P}$ et S) et/ou l'investigation approfondie d'une éventuelle zone suspecte.

Cette méthodologie est à opposer au phasage plus classique que nous avons utilisé jusqu'alors qui comprenait chronologiquement :

- une reconnaissance par sondages carottés;

- une campagne sismique essentiellement réalisée à partir des sondages, destinée en particulier à vérifier leur représentativité.

Le gros intérêt de la méthodologie proposée ci-avant est de mieux localiser les sondages et éventuellement d'en limiter le nombre.

\section{REMERCIEMENTS}

La publication du présent article a été rendue possible grâce à l'accord du Syndicat intercommunal du canal de la Bourne et de la commune de Chazelles-sur-Lyon, propriétaires des deux barrages décrits.

Les travaux du CEMAGREF en matière de sécurité des barrages sont encouragés par le ministère de l'Environ. nement (direction de l'Eau) et par le ministère de l'Agriculture (direction de l'Espace Rural et de la Forêt).

\section{BIBLIOGRAPHIE}

DEGOUTTE G., TOURMENT R. (1991), La santé des barrages anciens. Informations Techniques du CE. MAGREF, 81 (3), $7 \mathrm{p}$.

DEGOUTTE G., MONCHALIN G. (1986), Renforce. ment d'un barrage par des tirants actifs. Informations Techniques du CEMAGREF, 62 (3), $6 \mathrm{p}$.

LINO M. et al. (1991), Vieillissement de quelques bar. rages français très anciens. Pratique de leur réhabilitation. XVII ${ }^{2}$ Congrès CIGB Vienne (Autriche), Q. 65, R. 1, 1-21.

ENEL-ISMES (1991), The problem of dam ageing: ENEL experiences. XVII ${ }^{e}$ Congrès CIGB Vienne (Autriche), Q. 65, R. 36, 657.676.

BERTACCHI et al. (1991), Geophysical methods for the detection of ageing and effectiveness of repairs in dams. XVII ${ }^{\circ}$ Congrès $\mathrm{CIGB}$ Vienne (Autriche). Q. 65, R. 34, 619-636.

SEGG (1991), Auscultation sismique du barrage de la Gimond. Rapport d'études, Le Plessis-Robinson (France), $35 \mathrm{p}$. et annexes.

BERTRAND Y., BORDET C. (1982), Détermination in situ des propriétés dynamiques du rocher pour les projets de centrales nucléaires. Congrès international de l'Association internationale de Géologie de I'Ingénieur, New-Delhi (Inde).

BERTRAND Y., LAKSHMANAN J. (1985), Le Génie parasismique. Chapitre IV-6: «Mesures in situ des paramètres dynamiques du sol », Paris, Presses de l'ENPC.

BERTRAND Y., LAKSHMANAN J. (1987), Tomographies de vitesses sismiques en ondes de cisaille. ment. Journées de physique des laboratoires de l'équipement. 